IDIBELL, Barcelona, Spain; ${ }^{4}$ Royal Free London NHS Foundation Trust, University College London, London, UK

\subsection{6/thoraxjnl-2013-204457.178}

Background Although many randomised controlled trials (RCTs) and systematic reviews of treatment for latent tuberculosis (TB) infection (LTBI) have been conducted, previous analyses have not been able directly compare all utilised regimens. To address this we systematically searched for RCTs of LTBI treatment, then used a Bayesian network approach, which allows indirect headto-head comparisons, to determine the most efficacious regimens at preventing active TB and those that caused the fewest adverse events.

Methods PubMed, EMBASE and Web of Science were systematically mined using a search strategy developed to find RCTs of LTBI treatment. Animal studies, non-RCTs, and RCTs without at least one of our two endpoints were excluded. No language restrictions were made. Extracted data were inputted into a full random effects mixed treatment compartment model, based on code by Ades, Welton and Lu, and implemented in WinBUGS. Odds ratios for all possible comparisons in the network and hierarchical rankings for the different treatments were obtained from the model with point estimates taken as the median of the posterior distribution and 95\% credibility intervals (CrI) from the appropriate percentiles. Study quality was individually assessed.

Results 1,344 publications were generated by our search strategy, of which 52 fitted our criteria. 31 studies contained extractable data on adverse events and 44 on the development of active TB. 14 regimens were compared; an extract of the full results is presented (Table 1).

Conclusion Our Bayesian approach allows a novel, integrated, overview of the comparative efficacy and safety of different LTBI regimens, as well as a clear identification of the knowledge gaps where inference is difficult due to sparse data. The results of our study can therefore be used to inform guidelines and plan vital future LTBI treatment RCTs.

\begin{tabular}{|c|c|c|}
\hline \multirow[b]{2}{*}{ Regimen } & \multicolumn{2}{|c|}{ Ranking $(95 \% \mathrm{Crl})$} \\
\hline & Active TB & Adverse events \\
\hline Isoniazid $\leq 4$ months & $11(6,13)$ & $11(10,11)$ \\
\hline Isoniazid 6 months & $8(5,11)$ & $5(3,8)$ \\
\hline Isoniazid 9 months & $11(4,14)$ & $7(4,9)$ \\
\hline Isoniazid $\geq 12$ months & $6(4,9)$ & $7(4,9)$ \\
\hline Rifampicin/lsoniazid $\geq 3$ months & $7(4,10)$ & $4(3,7)$ \\
\hline
\end{tabular}

\section{P29 THE PATIENT'S PERSPECTIVE OF ANTI-TUBERCULOSIS TREATMENT}

CEJ Rang, N Sahota, A Jayaratnam; King George Hospital, Ilford, London, United Kingdom

10.1136/thoraxjnl-2013-204457.179

Background Tuberculosis (TB) requires prolonged antibiotic therapy with medications, which can cause a wide range of side effects. Despite this, it is essential that patients adhere to their treatment regimes to ensure treatment success and also to reduce the risk of transmission and of drug resistant TB developing. There is little in the literature regarding the patient's perspective of managing the substantial medication burden of TB treatment. However, to focus patient-centred care from local TB services and to direct future management of these patients, assessment of their perspective and quality of life is imperative.

Aim To assess patients who had completed anti-TB treatment over a 10 -month period as to their perspective regarding the TB service and treatment in a district general hospital.

Methods All patients who had completed anti-tuberculosis treatment over a 10 -month period were provided with an anonymous questionnaire. The data from these was collated and analysed.

Results 35 patients out of 64 patients completing TB treatment over a 10-month period returned the patient questionnaire. Of these patients $51 \%$ were female with an average age of 34 years. All the patients were aware how to contact the TB team and 94\% of patients received an information leaflet which they had read. $49 \%$ of patients suffered side effects; the most common being pruritis (23\%), rash (17\%), nausea (17\%), joint aches $(14 \%)$ and stomach upsets (11\%).

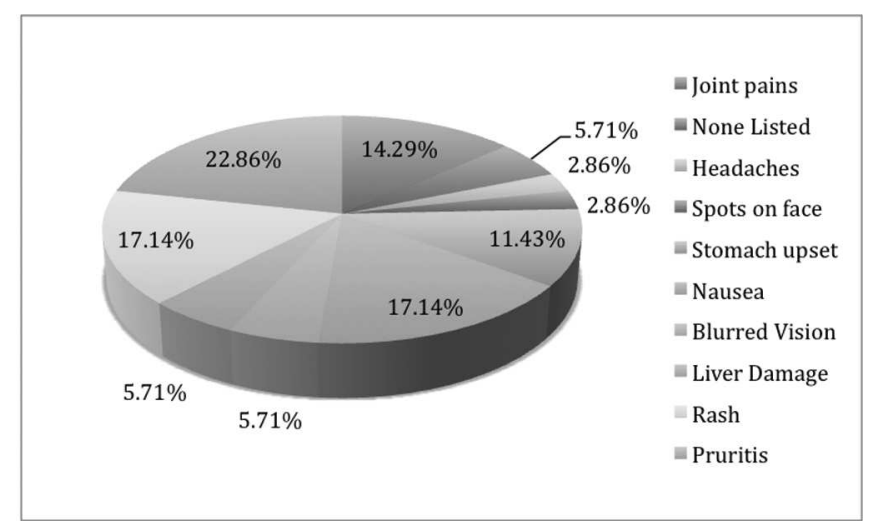

Abstract P29 Figure 1.

$86 \%$ of the patients felt that the support from the TB nurses did make a difference to their care. Overall patients found the ease of taking medications as follows: $40 \%$ very easy, $26 \%$ easy, $14 \%$ hard, $6 \%$ very hard, $11 \%$ neither easy or hard, $3 \%$ not answered.

Conclusion This study demonstrated that nearly half of the patients suffered from side effects whilst taking their treatment, impacting on their quality of life. Furthermore, a large proportion of patients $(20 \%)$ had difficulty taking their medications. However, patients do find that the TB service and the involvement of the TB specialist nurses have a positive impact on their care.

\section{P30 ARE WE UNDER USING DIAGNOSTIC INVESTIGATIONS IN THE MANAGEMENT OF ABDOMINAL TUBERCULOSIS (TB) IN HIGH ENDEMIC AREAS OF LONDON?}

C Stevens, S Sithamparanathan, T O'Shaughnessy; Newham University Hospital, London, United Kingdom

\subsection{6/thoraxjnl-2013-204457.180}

Introduction Abdominal tuberculosis presents with non-specific clinical symptoms. Diagnosis is based on clinical reasoning supported by radiological findings either by ultrasound or computer tomography. The gold standard of diagnosis remains with culture Mycobacteria tuberculosis complex. Therefore in areas with high 
endemic rates of $\mathrm{TB}$ are we under using investigations to gain tissues samples in suspected cases?

Method We undertook a retrospective analysis of 46 cases coded as abdominal tuberculosis from our local hospital register. Data was collected from case notes and computer systems regarding pathology and radiology results.

Results The majority of patients were born in the Indian subcontinent: India 39\%, Pakistan 20\% and Bangladesh 8\%. More than half the patients had lived in the UK for less than 5 years. $54 \%$ of patients had symptoms for 1-4 months before presentation. None of the cases had TB in the past and 13\% could recall possible TB contact.

$67 \%$ of CXR was normal, and of those who had abnormal films, only 4 cases had features that were specific for TB. Focused imaging taken included: CT abdomen 77\%, US abdomen $18 \%$, MR abdomen 1\%, Barium follow through 5\%.

$48 \%$ of patients had procedures to obtain histological and microbiological results, including laparoscopy, laparotomy, colonoscopy, gastroscopy or ascitic tap. $26 \%$ of patients underwent laparoscopy. Of the remaining patients, $18 \%$ obtained microbiological samples from alternative sites. Thus, $42 \%$ patients were treated on clinical symptoms and radiological image findings alone.

Discussion Laparoscopy has been regarded as the gold standard and diagnostic investigation of choice in the management of abdominal $\mathrm{TB}^{1,2}$. In our cohort $26 \%$ underwent laparoscopy. The reason for this unclear but could be due perceived risk with the procedure, lack of availability of service or in many cases is used as a last resort. In TB endemic areas, we suggest the development of an acceptable evidence based investigational pathway incorporating our surgical and gastrointestinal colleagues leading to more prompt and through management of abdominal tuberculosis.

\section{REFERENCES}

1. McLaughlin $S$, Jones $T$, Pitcher M, Evans P. Laparoscopic diagnosis of abdominal tuberculosis. Aust NZ I Surg 1998;68: 599-601

2. Rai S, Thomas W M. Diagnosis of abdominal tuberculosis: the importance of laparoscopy. J R Soc Med. 2003 December; 96(12): 586-588.

\section{P31 ASSESSING THE EFFECTIVENESS OF TUBERCULOSIS (TB) SCREENING IN NEW ENTRANT HEALTHCARE WORKERS USING DIFFERENT TIME CUT-OFFS TO DEFINE HIGH RISK INDIVIDUALS}

R Morton, K Bintley, Z Alexander, V Kahr; Epsom and St Helier University Hospitals NHS Trust, London, UK

\subsection{6/thoraxjnl-2013-204457.181}

Introduction and Objectives NICE (2011) and the Department of Health (2007) provide guidance for occupational health departments for TB clearance in healthcare workers. Previous work from the London Consortium of Occupational Health Providers (LCOHPS) shows a marked variation in practice, notably in the criteria for defining high risk individuals. The length of time in the UK for an individual from a high TB endemic area (defined as an incidence of 40 per 100,000 or greater) to be considered as low risk ranges from 6 months to $>5$ years. We performed a retrospective study of new trust employees to see if changing the definition of a high risk individual would impact on the effectiveness of our screening programme.

Methods We performed a retrospective study of 40 new employees at our trust between 2008 and 2012. Cases were selected on the basis of a positive QuantiFERON-TB Gold test at occupational health screening. Demographic data, including date of UK entry, were collected and analysed.

Results Results are summarised in Table 1.

Conclusions Changing the definition of a high risk individual by reducing the cut-off time since entry to the UK may have both financial and time-saving consequences. However, our data show that a significant proportion of healthcare workers with latent TB infection, and in some cases active TB infection, would be missed by reducing the cut-off to 1 year. Screening of healthcare workers is an important aspect in the prevention and control of TB. Reducing the effectiveness of this screening exposes patients to increased risk. In view of these data, we would not recommend reducing the cut-off time for the definition of a high risk individual to less than 5 years.

\begin{tabular}{|c|c|c|c|}
\hline \multirow{2}{*}{$\begin{array}{l}\text { Time since } \\
\text { entry to the } \\
\text { UK (years) }\end{array}$} & \multirow{2}{*}{$\begin{array}{l}\text { Number of } \\
\text { individuals } \\
(\%)\end{array}$} & \multicolumn{2}{|l|}{ Treatment received } \\
\hline & & Latent prophylaxis & Active TB treatment \\
\hline$<1$ & $11(27 \%)$ & 11 & 0 \\
\hline $1-5$ & $22(55 \%)$ & 18 & 2 \\
\hline$>5$ & $7(18 \%)$ & 6 & 1 \\
\hline
\end{tabular}

\section{P32 WHEN A TEST IS NEITHER POSITIVE NOR NEGATIVE: THE IMPACT OF EQUIVOCAL AND INDETERMINATE QUANTIFERON TB IGRA IN A UK POPULATION}

${ }^{1} \mathrm{P}$ Saunders, ${ }^{2} \mathrm{~J}$ Reading, 'J Harvey, ${ }^{3} \mathrm{~S}$ Murthy, ${ }^{3} \mathrm{~S}$ Capocci, 'S Hopkins, 'S Seneviratne, II Cropley, 'M Lipman; 'Royal Free London NHS Foundation Trust, London, United Kingdom; 'University College London Medical School, London, United Kingdom; ${ }^{3}$ University College London, London, United Kingdom

\subsection{6/thoraxjnl-2013-204457.182}

Introduction For the diagnosis of latent tuberculosis (LTBI), clinicians like interferon-gamma based assays (IGRA) to be either positive or negative. Tests can be indeterminate (failure of the positive or negative control); and recently an equivocal category has been introduced for QuantiFERON IGRA results that lie around the positive cut off, $0.35 \mathrm{IU} / \mathrm{mL}$, covering the range $0.2-$ $0.7 \mathrm{IU} / \mathrm{mL}$. Within our hospital, it is recommended that indeterminate or equivocal results are initially repeated by the requesting clinician. We report our outcomes from March 2010, when the equivocal category was introduced.

Methods Hospital pathology and clinical records were datamined. Cost analysis used local NHS costs.

Results Tests for 1964 individuals were processed (over onethird from Occupational Health, and another one-third pre-biological therapy). $92 \%$ of subjects had a definitive first result, with 6\% (116) equivocal and 2\% (42) indeterminate (Table 1). $60 \%$ of equivocal tests were below the positive cut off of 0.35 . The demographics of those with an equivocal result were broadly comparable with the whole tested population.

Almost half of the equivocal tests were not repeated (Table 1). $45 \%$ of repeats were negative and one-third still equivocal. 12 of 34 subjects referred to the TB service were treated for LTBI-10 with a positive IGRA on re-testing. One other patient with an initial equivocal test developed active TB during follow up.

$43 \%$ of people with an indeterminate result had no repeat test (Table 1). 1 of 6 subjects referred to the TB service following an indeterminate IGRA received LTBI treatment. 


\section{Correction}

C Stevens, S Sithamparanathan, T O'Shaughnessy. Are we under using diagnostic investigations in the management of abdominal tuberculosis (tb) in high endemic areas of london? Thorax 2013;68(Suppl 3):A87. doi:10.1136/thoraxjnl-2013-204457.180.

In the results section of this abstract, the sentence should read: 'Thus, $34 \%$ of patients were treated on the basis of clinical symptoms and radiological image findings alone.'

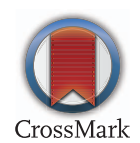

Thorax 2014;69:98. doi:10.1136/thoraxjnl-2013-204457.180corr1 